\title{
Endocrine regulation of predator-induced phenotypic plasticity
}

\author{
Stuart R. Dennis · Gerald A. LeBlanc • \\ Andrew P. Beckerman
}

Received: 9 April 2014 / Accepted: 17 September 2014 / Published online: 5 October 2014

(C) The Author(s) 2014. This article is published with open access at Springerlink.com

\begin{abstract}
Elucidating the developmental and genetic control of phenotypic plasticity remains a central agenda in evolutionary ecology. Here, we investigate the physiological regulation of phenotypic plasticity induced by another organism, specifically predator-induced phenotypic plasticity in the model ecological and evolutionary organism Daphnia pulex. Our research centres on using molecular tools to test among alternative mechanisms of developmental control tied to hormone titres, receptors and their timing in the life cycle. First, we synthesize detail about predator-induced defenses and the physiological regulation of arthropod somatic growth and morphology, leading to a clear prediction that morphological defences are regulated by juvenile hormone and life-history plasticity by ecdysone and juvenile hormone. We then show how a small network of genes can differentiate phenotype expression between the two primary developmental control pathways in arthropods: juvenoid and ecdysteroid hormone signalling. Then, by applying an experimental gradient of predation risk, we show dose-dependent gene expression linking predator-induced plasticity to the juvenoid hormone pathway. Our data support three conclusions: (1) the juvenoid
\end{abstract}

Communicated by Jennifer Thaler.

S. R. Dennis $(\bowtie) \cdot$ A. P. Beckerman

Department of Animal and Plant Sciences, University

of Sheffield, Western Bank, Sheffield, UK

e-mail: stuart.dennis@sheffield.ac.uk

A. P. Beckerman

e-mail: a.beckerman@sheffield.ac.uk

G. A. LeBlanc

Environmental and Molecular Toxicology Program, Department of Biological Sciences, North Carolina State University, Raleigh, NC 27695-7633, USA signalling pathway regulates predator-induced phenotypic plasticity; (2) the hormone titre (ligand), rather than receptor, regulates predator-induced developmental plasticity; (3) evolution has favoured the harnessing of a major, highly conserved endocrine pathway in arthropod development to regulate the response to cues about changing environments (risk) from another organism (predator).

Keywords Phenotypic plasticity - Daphnia pulex . Juvenile hormone $\cdot$ Gene expression · Developmental control · Predation risk

\section{Introduction}

Phenotypic plasticity - the expression of different phenotypes in different environments by single genotypes-can alter the mean and variance of traits on which selection can act. This topic draws the attention of ecologists interested in the origins and consequences of trait variation, of evolutionary biologists interested in plasticity as a source of selectable variation, and of developmental biologists interested in genes, hormones and the developmental control of traits such as morphology and life history (Nijhout 2003b; Pigliucci 2001; Sultan 2007; Tollrian and Harvell 1999). Identifying the role of development and physiology in the expression of plasticity is central to defining the mechanisms that underpin trait variation. Strong links between phenotypic plasticity and developmental biology are established in plants, where it is understood how endocrine physiology mediates environmental signals to produce phenotypes. Plant biology long ago embraced a molecular physiology-ecology agenda to link population and physiological and molecular ecology (e.g. Baldwin et al. 2001; Weston et al. 2008). In contrast, developmental 
and physiological explanations for phenotypic plasticity in animals are much less frequent and less developed (for a recent review, see Flatt and Heyland 2011).

There are several animal examples detailing how endocrine signalling can mediate single trait responses to abiotic features of the environment (Table 1). Classic examples centre on arthropods and their two major developmental hormones-juvenile hormones and ecdysteroids. They include examples of endocrine control of polymorphism such as seasonal colour variation in butterfly wings (Rountree and Nijhout 1995) and temperature regulation of colour morph in Manduca sexta (Truman et al. 1973). They also include examples of the endocrine control of threshold traits (on-off; polyphenism) as examples of plasticity including butterfly wing patterning (Brakefield et al. 1998), sexual ornaments in horned beetles (Emlen and Nijhout 1999) and temperature-dependent sex determination in Daphnia (Olmstead and LeBlanc 2007). The central feature of these examples is a response of the juvenoid and ecdysteroid hormone signalling pathways to an abiotic environmental stress, producing change in a single trait. These examples (Table 1) provide valuable evidence that polymorphisms and polyphenisms are under endocrine control. Here, we extend such efforts to reveal endocrine control of phenotypic plasticity in response to another organism-the case of predator-induced phenotypic plasticity.

Predator-induced plasticity (i.e. predator-induced defences) has emerged across many taxa as an example of continuous phenotypic plasticity, despite years of being considered an on-off, threshold trait (Roff 2002). The plasticity is typically detected in three classes of traits: morphology, life history and behaviour. The ecological responses of freshwater vertebrates and invertebrates to predator cues have been well studied (e.g. Beckerman et al. 2010; Dennis et al. 2011; Hammill et al. 2008; Hoverman and Relyea 2009, 2001b; Riessen 1999; Tollrian 1995b; Tollrian and Harvell 1999). Key findings from cladocera and rotifera (for reviews see Lass and Spaak 2003; Tollrian and Dodson 1999), and anuran and odonate species (e.g. Laurila et al. 2002; Relyea 2001a; Van Buskirk 2002) indicate that predator-induced defences are continuous, multivariate, adaptive, confer a survival benefit, alter the distribution of populations and species, and can substantially influence population dynamics. The reaction norms for these induced defences (responses) are also rarely as steep as a simple polyphenism definition would suggest (see Dennis et al. 2011; Hammill et al. 2008).

The predator-induced variation in the timing of the life cycle and morphology that defines predator-induced plasticity indicates that it is likely a function of variation in the temporal and spatial regulation of development. This system thus offers one of the richest possibilities for extending our understanding about how endocrine physiology mediates developmental control of phenotypic plasticity, extending previous efforts limited to single trait responses to abiotic stress (Table 1). Here we provide a functional explanation of how predator-induced phenotypic plasticity is generated.

Table 1 Examples of endocrine-mediated polyphenisms (trait), the identified mechanisms and their environmental cues

\begin{tabular}{|c|c|c|c|c|}
\hline Organism & Polymorphism & Mechanism & Cue & References \\
\hline Butterflies & $\begin{array}{l}\text { Hindwing melanism and } \\
\text { eyespot size } \\
\text { Eyespot presence }\end{array}$ & $\begin{array}{l}\text { Ecdysteroid timing and } \\
\text { duration } \\
\text { Ecdysteroid receptor } \\
\text { expression }\end{array}$ & $\begin{array}{l}\text { Seasonal: photoperiod } \\
\text { Temperature } \\
\text { Seasonal: photoperiod } \\
\text { Temperature }\end{array}$ & $\begin{array}{l}\text { Beldade and Brakefield (2002), } \\
\text { Brakefield et al. (1998), Endo } \\
\text { and Kamata (1985), Rountree } \\
\text { and Nijhout (1995), Sawada } \\
\text { et al. (2002) }\end{array}$ \\
\hline Manduca sexta larvae & Larval colour green/black & JH titre & Temperature & $\begin{array}{l}\text { Safranek and Riddiford (1975), } \\
\text { Suzuki and Nijhout (2006) }\end{array}$ \\
\hline Onthophagus beetles & $\begin{array}{l}\text { Horn growth } \\
\text { Horn size }\end{array}$ & $\begin{array}{l}\text { Ecdysteroid pulse } \\
\text { JH titre }\end{array}$ & Body size & $\begin{array}{l}\text { Emlen and Nijhout (1999), } \\
\text { Moczek and Emlen (1999) }\end{array}$ \\
\hline Daphnia spp. & Sex determination & JH titre & $\begin{array}{l}\text { Seasonal: photoperiod } \\
\text { Temperature } \\
\text { Nutrition } \\
\text { Pop density }\end{array}$ & $\begin{array}{l}\text { Hebert (1978), Olmstead and } \\
\text { Leblanc (2002), Tatarazako } \\
\text { et al. (2003) }\end{array}$ \\
\hline Crickets & Wing length & $\begin{array}{l}\text { JH titre } \\
\text { Ecdysteroid titre }\end{array}$ & $\begin{array}{l}\text { Temperature } \\
\text { Photoperiod } \\
\text { Diet } \\
\text { Pop density }\end{array}$ & $\begin{array}{l}\text { Zera and Bottsford (2001), } \\
\text { Zera and Denno (1997) }\end{array}$ \\
\hline Termites & Caste differentiation & JH titre & Pheromone & $\begin{array}{l}\text { Hartfelder and Emlen (2005), } \\
\text { Zhou et al. (2007) }\end{array}$ \\
\hline Aphids & Winged forms (alates) & JH titre & $\begin{array}{l}\text { Seasonal: photoperiod } \\
\text { Temperature } \\
\text { Pop density }\end{array}$ & $\begin{array}{l}\text { Hardie (1980), Hardie et al. } \\
\text { (1985) }\end{array}$ \\
\hline
\end{tabular}


Specifically, we show that predator-induced prey morphological defences in Daphnia pulex are regulated in a dose-dependent manner by the juvenoid hormone signalling pathway, which, along with ecdysteroids, form the major endocrine signalling pathways in most arthropods. Specifically, we show that evolution has favoured the harnessing of a major, highly conserved endocrine pathway in arthropod development to regulate the response to cues about changing environments (risk) from another organism (predator).

We present three sets of data central to this result. First, we present a concise and novel synthesis of predation and the physiological regulation of arthropod somatic growth and morphology, leading to a clear prediction that morphological defences are regulated by juvenile hormone and lifehistory plasticity by ecdysone and juvenile hormone. Second, we present a small gene network dominated by highly conserved nuclear receptors (Escriva et al. 2004) that can, via RT-qPCR, be used to distinguish between activity of the juvenile hormone and ecdysone signalling pathways under experimental conditions. Finally, we present experimental data on gene expression using this network to clearly show that predator-induced prey morphological defences in Daphnia pulex are regulated in a dose-dependent manner by the juvenile hormone pathway.

\section{Methods}

Our methods consist of four steps. First we document, via a synthetic review of the arthropod developmental biology literature, that inducible defences in arthropods must be under hormonal control by the juvenoid or ecdysteroid hormone signalling pathway. In contrast to recent work focusing on neurotransmitters (Weiss et al. 2012), we focus our study on endocrine regulation. Environmental signals received by sensory apparatus are transmitted, usually via neurotransmitters and the nervous system, to the endocrine system, which then orchestrates appropriate phenotypic responses in target tissues. The effective integration of all these processes is common and important but the endocrine system is always required for phenotypic plasticity in life history and morphology, whereas neurotransmitters alone are sometimes sufficient for behavioural change.

Second, via the same arthropod development literature, we document a small gene network capable of distinguishing, via RT-qPCR, the activity of either pathway under experimental conditions. Third, we use this network via RT-qPCR and experimental exposure of daphnids along a gradient of predation pressure to reveal which pathway is mediating the plastic response to predation risk.
Study system

We use Daphnia pulex, a model organism for ecological interactions, ecotoxicology, arthropod developmental biology and environmental genomics (Colbourne et al. 2011). It is a common freshwater crustacean (water flea) that experiences predation risk from the phantom midge Chaoborous flavicans. The midge predator produces a low molecular weight kairomone that induces changes in D. pulex life history and morphology (Tollrian and Von Elert 1994). Specifically, C. flavicans induces later age and larger size at maturity as well as prominent neckteeth during the second and third instars of development, corresponding to the age (size) classes most sensitive to midge predation (see Fig. 1 and Tollrian 1995a). There is extensive genetic variation in these responses (i.e. genetic variation in plasticity), and the responses are adaptive (Beckerman et al. 2010; Hammill et al. 2008; Tollrian 1995b). For example, the induced neckteeth can increase survival by up to $45 \%$ (Hammill et al. 2008). These ecological patterns and decades of research make $D$. pulex a model ecological example.

Physiological review and discovery of the gene network

We investigated the arthropod literature on the regulation of somatic growth and morphology by juvenoid and ecdysteroid signalling in arthropods. These data provide detailed information on how the regulation of development by the endocrine system may determine patterns of growth, development and morphology under predation risk. These data also identify a gene network capable of distinguishing, via RT-qPCR, the activity of either pathway under experimental conditions.

Kairomone exposure to reveal juvenoid or ecdysteroid control

In order to discriminate between juvenoid and ecdysteroid control of predator-induced plasticity using the gene network, $D$. pulex were exposed to predator chemical cues along a gradient of increasing predation risk and serially sampled to isolate RNA.

Daphnids were routinely maintained in hard artificial pond water (ASTM 2007), and fed the algae Chlorella vulgaris at $21{ }^{\circ} \mathrm{C}$ in controlled-temperature rooms on a $16: 8$ light cycle. We extracted kairomone from frozen Chaoborus flavicans (Honka, Germany), following the method developed by Tollrian (see also Hammill et al. 2008; Tollrian 1995b).

To produce a gradient of predation risk, we exposed replicate individuals independently to four concentrations of extracted predator cue $\left(0,0.1,0.5,1 \mu \mathrm{L} \mathrm{mL}^{-1}\right)$. 
Fig. 1 Exposure to chemical cues from midge (Chaoborus flavicans) larvae results in a defended phenotype in Daphnia pulex. a Second-instar daphnid exhibiting a defended morphology. b An undefended secondinstar daphnid

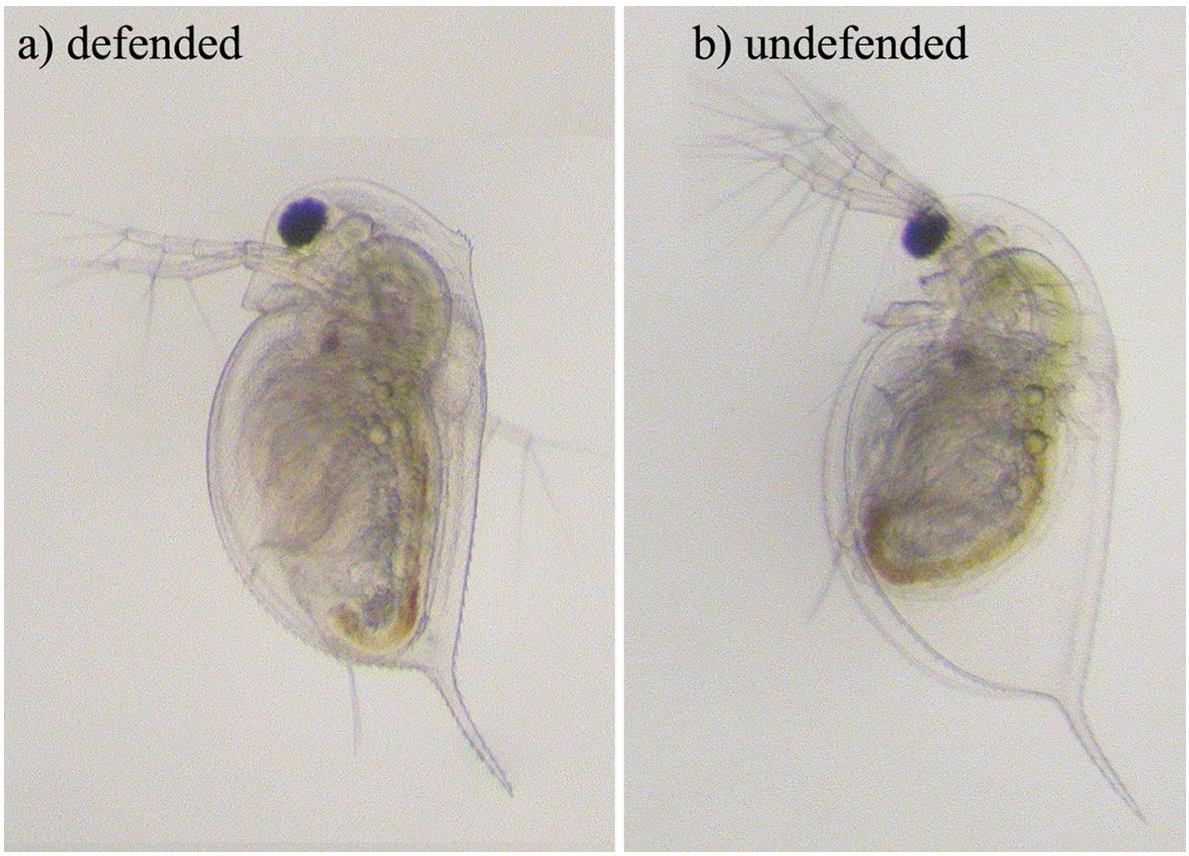

Gene expression at release from brood pouch
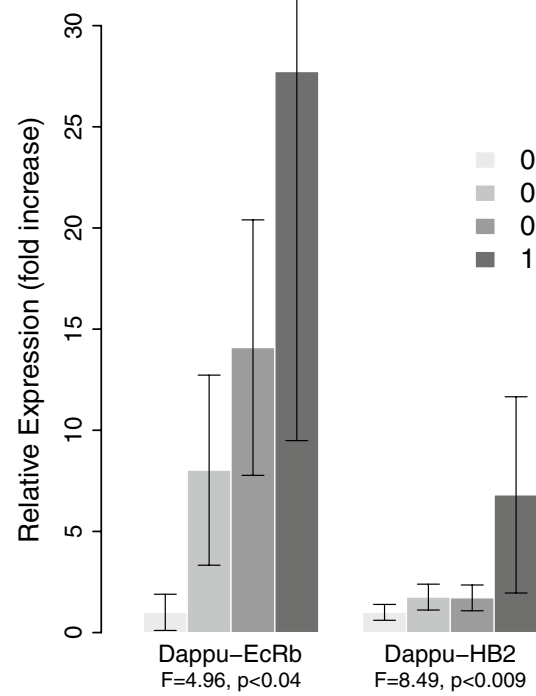

$0 \mu \mathrm{l} / \mathrm{ml}$ (control)

- $0.1 \mu \mathrm{l} / \mathrm{ml}$

$0.5 \mu \mathrm{l} / \mathrm{ml}$

- $1 \mu \mathrm{l} / \mathrm{ml}$
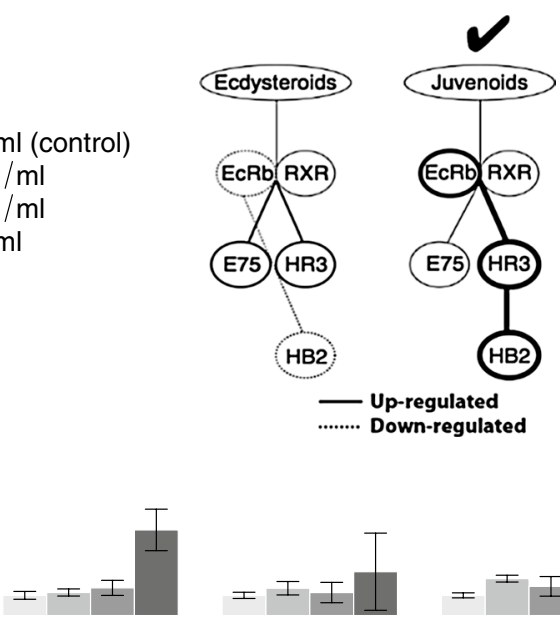

Dappu-HR3

$F=28.9, p<0.0001$

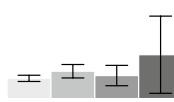

Dappu-E75

$F=1.23, p=0.27$

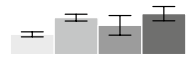

Dappu-RXR

$\mathrm{F}=2.6, \mathrm{p}=0.12$
Fig. 2 Relative expression of the five genes in the proposed mini gene network. Significant predator kairomone dose-dependent upregulation of Dappu-EcRb, Dappu-HR3 and Dappu-HB2 expression are consistent with an increase in juvenoid hormone titres. Inset: a fivegene network, dominated by nuclear receptors central to arthropod

For each treatment, 60 third-generation mothers who had finished their second brood were each exposed to the relevant concentration of chemical cue. Exposures began when thirdbrood embryos developed eyespots (approximately $24 \mathrm{~h}$ prior to release from the brood chamber). Each jar was checked development, that can discriminate between activity in the ecdysteroid and juvenoid endocrine pathways. Solid lines indicate upregulation (more expression) and dashed lines indicate downregulation (less expression). Thicker vs. thinner lines indicate relative (qualitative) magnitudes of gene expression

hourly, and neonates (10-15) were collected at the time of brood pouch release. Neonates from three sets of 20 mothers for each treatment were pooled in RNALater for subsequent RNA extraction. We focused on expression at brood release because it is in the middle of the established perinatal time 
course of induced morphological change: embryo exposure to predator kairomones results in substantial morphological expression of neckteeth (Fig. 2a) on a pedestal at instar two, a time delay of $\sim 3-4$ days, with release of neonates from the brood pouch being in the middle (Laforsch and Tollrian 2004; Naraki et al. 2013; Parejko 1992).

\section{RNA isolation and cDNA conversion}

Samples were homogenized and RNA isolated from the homogenate using the SV Total RNA Isolation System (Promega), following the manufacturer's instructions. RNA yield and purity were determined by absorbance $(260 \mathrm{~nm}$ and 260/280 $\mathrm{nm}$ ratio, respectively) using a Nanodrop ND-1000 spectrophotometer. RNA was reverse-transcribed to cDNA with random hexamers and oligo DT primers using the ImProm-II Reverse Transcription system (Promega) and/or the HighCap RT kit (Applied Biosystems), following the manufacturer's instructions.

\section{Primer design}

Genes were identified via our review and synthesis (see below). Primers for $\beta$-actin, EcRb, RXR, E75, HR3 and HB2 were designed using Primer3 and Amplify $3 X$ (v3.1.4) after locating EST libraries of the genes from wFleabase. Primers were developed as follows. Blast searches against the genes from other organisms and contigs were assembled using CAP3. Contigs were then blasted against the Daphnia pulex genome. The resulting exon-exon spanning primers were: $\beta$-actin forward (f)-TGGTCAGGTCATCA CCATTG, reverse (r)-CTCGTGGATACCGCAAGATT; DappuEcRb f-TCGTCATCTCGGTCATGTGT, r-TGAACT ACCCTCCGAAGACG; DappuRXR f-GTTCAAGAGGA GAGGCAACG, r-AATCACTGGTGGCATCCATATC; DappuE75 f-CACTGGTTCCAATTGCTTTG, r-GTCTCG ATCGTAACGTCTTGC; DappuHR3 f-GGGCGTCCA TTATGGAGTCA, r-CGGAAGAAACCCTTGCAGC; DappuHB2 f-CAAAGTCCTCCTCCCAAGC, r-CTGTTGG GCAACGTCAACTA.

rt-QPCR (quantitative real-time PCR) was performed (in procedural triplicate) with an Applied Biosystems Step-One real-time PCR machine using default parameters. Amplification mixtures consisted of $2.5 \mu \mathrm{L}$ SYBR Green PCR Master Mix (Applied Biosystems), $10 \mathrm{nM}$ primers, and $250 \mathrm{ng}$ template cDNA in a total volume of $5 \mu \mathrm{L}$. Reactions were held at $95{ }^{\circ} \mathrm{C}$ for $10 \mathrm{~min}$, followed by 40 cycles of $95^{\circ} \mathrm{C}$ for $15 \mathrm{~s}$ followed by $60^{\circ} \mathrm{C}$ for $1 \mathrm{~min}$. At the end of cycling, the melting temperatures of PCR products were determined to ensure no amplification of non-target DNA. The comparative CT method (Cy0) was used to assess the relative levels of EcRb, E75, HB2, HR3 and USP normalized to mRNA levels of $\beta$-actin measured with the same sample.

\section{Results}

Endocrine control of predator-induced plasticity: a mini-review and synthesis

There are several independent lines of published evidence that, when combined, predict that predator-induced phenotypic plasticity in morphology and life history is under endocrine regulation by juvenoid and ecdysteroid signalling. The evidence comes from detailed information on why development matters under predation risk and how the endocrine system regulates growth, development and morphology in arthropods. In the following paragraphs we offer a concise review of this evidence.

\section{Why development matters under predation risk}

Life history (e.g. size and age at maturity) and morphology (e.g. defence structures) are two of the three most common classes of traits that respond to predation risk (the third is behaviour). In vertebrates and invertebrates, adaptive changes of $> \pm 10 \%$ in the size and age at maturity and growth rate are not uncommon (Abrams and Rowe 1996; Benard 2004; Peckarsky et al. 2002; Relyea 2001a; Teplitsky et al. 2005). In arthropods (i.e. moulting organisms), such changes in the timing of the life cycle can only occur by shifting the number of, or the duration between, moults.

There are also particularly dramatic responses to predation risk where predator chemical cues induce de novo morphological changes in either shape, colour or the appearance of protuberances (Hoverman and Relyea 2009; Lass and Spaak 2003; Relyea 2001a, b; Tollrian 1995b). The same prey species can often show increases and decreases in size at maturity, age at maturity and growth rate, depending on whether they are exposed to large- or small-sized selective predators (Beckerman et al. 2010).

Such changes in the timing of maturation/metamorphic events and in morphology require major alternations of the physiological processes that regulate somatic growth and development-processes that are well understood in vertebrates and invertebrates. In arthropods (the focus of this study), the timing and spatial patterning of development are regulated by the endocrine system and specifically by the juvenoid/ecdysteroid signalling pathway (see Flatt and Heyland 2011).

\section{Endocrine mediation of growth, development and morphology in arthropods}

Evidence from a wide range of arthropod species indicates that two hormones oversee the developmental regulation of the timing of moults and the inter-moult duration as well as the transition from juvenile to reproductive 
adult: juvenile hormone and ecdysteroids (e.g. Cruz et al. 2003; Davidowitz and Nijhout 2004; Flatt and Heyland 2011; Gelman et al. 2002; Riddiford 1993; Riddiford et al. 2003; Truman and Riddiford 2007). Furthermore, research on major morphological changes in insects identifies juvenile hormones as the major regulating factor (e.g. Berger and Dubrovsky 2005; Chang et al. 1993; Hall 1999), either directly as a transcriptional regulator of target genes or indirectly through suppression or augmentation of ecdysteroiddependent transcriptional regulation (Berger and Dubrovsky 2005; Jindra and Riddiford 1996). Essentially, existing data underpin and spawn our hypothesis that morphological responses to predators in arthropods should be under the control of juvenile hormone, and that life-history responses to predators should be under the control of the interplay between ecdysteroid regulation of the moult and juvenile hormone limitation of maturation. This established developmental detail, combined with knowledge of the continuous nature of predator-induced phenotypic plasticity in D. pulex and other arthropods, suggest the following formal prediction: kairomones-external cues of risk from predatorsalter phenotypes by mobilising and mediating ecdysteroid and juvenoid hormone and hormone receptor expression in a dose-dependent manner, facilitating fine-scale, adaptive developmental changes in phenotype to predation risk.

How to discriminate between juvenoid and ecdysteroid signalling

The previous section defined our hypothesis that predatorinduced phenotypic plasticity in arthropods should be controlled by the juvenoid and/or ecdysteroid signalling pathways. The literature on arthropod developmental control also reveals that gene expression patterns in a small gene network (Fig. 2), dominated by nuclear receptors, can discriminate between whether the juvenoid or ecdysteroid pathway is correlated with phenotypic plasticity. In addition to supplying a formal tool for investigating physiological regulation of plasticity, we show below that this gene network can also help identify the developmental control mechanism (i.e. changes in receptor or ligand expression profiles, see Nijhout 1999) underpinning this activity.

This network (Fig. 2) comprises five genes (referenced here to the Daphnia pulex genome by the prefix "Dappu-") with orthologues throughout the arthropods: the two components of the nuclear receptor heterodimer for ecdysteroids, Dappu-EcR and Dappu-RXR, and the "early" response genes Dappu-HR3, Dappu-E75 and Dappu-HB2 (a haemoglobin gene). Four of these five gene products (the exception being HB2) are essential for moulting and metamorphosis in Tribolium (Tan and Palli 2008) and probably all arthropods (Erezyilmaz 2011; Heyland et al. 2011; King-Jones and Thummel 2005).
Figure 2 shows endocrine pathway specific expression patterns for ecdysteroid or juvenoid action. We derived these pathway-specific patterns of expression from the following detail. Upregulation of HR3 indicates involvement of the ecdysteroid and/or juvenoid pathways without discrimination between them, as both pathways elicit a positive response (Hannas and LeBlanc 2010). In several taxa, E75 is unresponsive to juvenoids but upregulated by ecdysteroid pathway activation (Hannas and LeBlanc 2010; Soin et al. 2008). Moreover, E75 expression is coincident with ecdysteroid pulses in many other insects (King-Jones and Thummel 2005; Sullivan and Thummel 2003), is known to cycle its expression in response to ecdysteroids during the moult cycle in both insects and crustaceans (Priya et al. 2010; Siaussat et al. 2004), and is a repressor of HR3 activity (Hannas et al. 2010). Its precise role during moulting in daphnids remains unclear, but we include it in our profiling due to its regulation of HR3 activity (Hannas et al. 2010).

In Tribolium, the haemoglobin gene HB2 is upregulated by juvenoids and downregulated by ecdysteroids (Parthasarathy and Palli 2009). Similarly, HB2 expression can be induced by juvenoids in both $D$. magna and $D$. pulex (Dm-HB2 and Dappu-HB2, respectively) (Rider et al. 2005). If the same pattern (downregulation by ecdysteroids) is true of Dappu-HB2, expression changes in Dappu-HB2 would provide discrimination between the two (juvenoids and ecdysteroids), with the caveat that oxygen tension may also alter the haemoglobin dissociation curve (Lambertsen et al. 1952) and therefore expression of Dappu-HB2. In this context, therefore, Dappu-HB2 may only be an appropriate indicator for laboratory studies. In D. magna, EcRb (Dm$\mathrm{EcRb} ; \mathrm{Dm}=D$. magna) is downregulated by ecdysteroids and upregulated by juvenoids, while expression of its heterodimeric partner (Dm-RXR) is unaffected by either class of hormone. In insects, juvenoid mimics (e.g. methoprene) affect expression of EcRb variably: expression is marginally upregulated at time points distal to ecdysis and downregulated by juvenoids near ecdysis (see Parthasarathy and Palli 2009).

These details combine to allow the gene expression pattern based discrimination of developmental pathways (Fig. 2). They provide a diagnostic tool for experimental evaluation of how environmental stress such as predation is captured by developmental processes to generate phenotypic plasticity. In the next two sections, we use this gene network to reveal the juvenoid pathway regulation of predator-induced plasticity.

Gene expression and pathway identification

We exposed $D$. pulex to a gradient of four midge kairomone concentrations (control + three concentrations; see "Methods") and used RT-qPCR to assess expression 
pattern in the Fig. 2 gene network along this gradient. The discriminatory power of our network revealed which hormone pathway likely regulates predator-induced plasticity: juvenoids.

At brood release, we detect a correlated and very clear, predator-cue, concentration-dependent pattern of relative gene expression consistent with juvenile hormone action (Fig. 2). The same four kairomone concentrations also produce a sigmoid and increasing expression of morphological defence at second instar (see Dennis et al. 2011). Three genes, Dappu-EcRb, Dappu-HR3 and Dappu-HB2, are upregulated in a kairomone-dose-dependent manner relative to control conditions. The remaining two genes, Dappu-RXR and Dappu-E75, are neither up- nor downregulated. This pattern of expression is most coincident with the juvenoid expression profile (Fig. 2, inset). Our conclusion is made more robust by the absence of Dappu-E75 upand downregulation.

\section{Discussion}

By combining an experiment with $D$. pulex exposed or not exposed to Chaoborus kairomone with expression profiles from a small gene network capable of discriminating between developmental control hormone action, we have shown that juvenile hormone signalling is strongly implicated in predator-induced plasticity in a predator cue, dosedependent manner (Fig. 2).

Our data demonstrate that an environmental signal (kairomone) received during embryonic development is able to co-opt juvenoid signalling for de novo production of a defensive structure. Juvenoid control of morphological change is well established in many arthropods (e.g. Cruz et al. 2003; Davidowitz and Nijhout 2004; Gelman et al. 2002; Riddiford 1993; Riddiford et al. 2003; Truman and Riddiford 2007). Decades of insect research shows that the titre of juvenile hormone influences the phenotype in the next developmental stage.

Several workers have shown that juvenoids can influence daphnid morphology. Oda and colleagues (2011) exposed D. galeata to increasing concentrations of methyl farnesoate and fenoxycarb (pesticide juvenile hormone analogue), both of which generated allometric shifts in the relationship between head shape and body size. Furthermore, Miyakawa and colleagues (Miyakawa et al. 2013) have shown that $\mathrm{JH}$ analogues may change the perception of the cue, but they and we (Dennis and Beckerman, unpublished data) find no evidence that $\mathrm{JH}$ can directly induce neckteeth de novo in $D$. pulex. This indicates that the cue for de novo defence production is not the hormone $(\mathrm{JH} / \mathrm{MF})$, but the cue triggers variation in the endocrine control of morphology, presumably via JH interaction.
As we noted in the "Introduction", changes in the timing of life-cycle events and morphology, hallmarks of predatorinduced plasticity, require major alternations of the physiological processes that regulate somatic growth and development—processes that are well understood in vertebrates and invertebrates. Decades of fundamental physiological research have shown that the juvenoid and ecdysteroid hormones and signalling pathways are responsible for such regulation in the arthropods (Flatt and Heyland 2011; Riddiford 2008; Riddiford et al. 2003). Furthermore, evidence from vertebrates and nematodes such as $C$. elegans suggest that there may be similar core developmental pathways regulating development in most animals. This offers the tantalising insight that there are, at least in the arthropods, core and quite conserved developmental processes that interface with the internal and external environment to shape development (Flatt and Heyland 2011) and thus facilitate phenotypic plasticity, which is so often centred on variation in the timing of life-cycle events and morphology. As Flatt and Heyland (2011) reveal throughout their book, linking core developmental processes to peripheral cellular and physiological processes will provide the insight necessary to understand sources of variation and potentially targets of selection in life history and morphology.

\section{Developmental control of predator-induced plasticity}

The patterns of expression in the gene network (Fig. 2) further allow us to reconstruct details about the developmental control mechanism for this dose-dependent effect of juvenile hormone. Developmental biologists suggest that changes in somatic growth and development in insects is controlled by variation in four features of endocrine regulation (Nijhout 1999, 2003a, b): the titre, threshold, timing and sensitive period. The titre is the amount of a given signal (e.g. ligand: hormone/peptide). The effect of this titre depends on whether the amount exceeds a threshold. The action of the hormone involves not only surpassing a threshold, but doing so with timing that overlaps with a sensitive period defined by the receptor. An adjustment or change in any one of these four endocrine traits, as generated by an external cue, represents a mechanism of developmental control and a method by which adaptive plasticity may arise in response to an environmental cue (Nijhout 1999, 2003b). Furthermore, the amount of overshoot of the threshold by the titre, the accuracy of the timing and sensitive period, and the titre of the receptor provide mechanisms by which the phenotypic plasticity can be characterised as a smooth reaction norm, rather than a discrete threshold trait.

Our data show a predator cue generated dose-dependent response in the major components of the juvenoid expression pathway. Combined with previous data on dose-dependent 
morphological profiles (see Dennis et al. 2011; Hammill et al. 2008), our data indicate that the developmental control mechanism resides in a change in the titre of juvenoids, not a change in the threshold, timing or sensitive period. Specifically, in insects, E75 expression is coincident with ecdysteroid pulses (King-Jones and Thummel 2005); because Dappu-E75 expression is neither up- nor downregulated compared to controls, we can eliminate ecdysteroid titre changes between control and predator environments. Similarly, as Dappu-RXR expression is unchanged, the EcR/RXR receptor expression appears unchanged; the upregulation of Dappu-EcRb is likely a result of juvenoid hormone exposure, especially as neonates are far from ecdysis at this time (see above). The upregulation of Dappu-HR3 and DappuHB2 also suggest juvenoid-like titre activity.

RT-qPCR and the detection of endocrine function

Our gene network reference tool, comprising highly conserved nuclear receptors, offers a compelling alternative to more common methods of assaying hormone titres directly (e.g. radio-immunoassays), and one that has many benefits. First, the amount of biological and consumable material necessary to query the gene network is much less than the amount of haemolymph and consumables needed to use the most advanced tools for radio-immunoassays (RIA), HPLC, mass spectroscopy, etc. Importantly, the low biological material requirements make it possible to query patterns in very small arthropods, which do not typically provide sufficient material for traditional methods. Second, the gene network provides a much more direct route to making inferences about developmental control mechanisms (see above), augmenting typical inferences from RIA and HPLC methods. To gain similar insight into developmental control mechanisms, RIA methods would require assays of multiple genotypes, each with different levels of phenotypic expression, as these methods only measure titres. While exploring variation in multiple genotypes is valuable in its own right, our approach indicates that it is not needed to be able to makes inferences about the mechanisms of developmental control.

The future of target gene approaches in the genomic era

Rapid advances in multi-omics approaches will soon supersede single/few-target techniques such as RT-qPCR and panel approaches such as microarrays. Falling costs and high-capacity "next-generation sequencing" mean that already it is economically viable and practical to capture an organism's entire transcriptome simultaneously using RNAseq, rather than a select few targets or microarray. This transcriptome may at first seem attractive, and indeed from a data-generation perspective it is very attractive, but there are many pitfalls, and we advise a degree of caution in the use of such data-dense methods. First, there is a naïve assumption that all parts of the genome will be sequenced with the same probability, governed only by their initial starting abundance, and that that abundance will be proportional in the sequenced dataset. Unfortunately, we already know that this is simply not true (Finotello et al. 2014; Lauss et al. 2013; Tuller 2014). Whereas RT-qPCR ensured "equality" through reaction optimization by designing specific (exon-exon junction spanning) primers for transcripts of interest, and matching amplification efficiencies, amplicon length and GC content, whole-transcript suffers from inherent biases in sequencing due to transcript length (Oshlack and Wakefield 2009), composition and overrepresentation of highly abundant transcripts (Young et al. 2010).

Second, genomes are rich with tens of thousands of genes, all of which can covary in concert and may depend on complicated coexpression and redundancy. Further, small changes in receptor occupancy (as little as $10 \%$ ) can have profound biological effects, or none. Therefore, subtle changes in expression that are biologically meaningful or gross changes in expression without biological significance can be incorrectly estimated, especially when many thousands of tests are conducted: a biologically significant relationship may be found or missed purely by chance.

Together, these sequencing biases and type I and II errors combine to suggest a set of tools that are required when using genomic data to ask the types of questions we focus on here. Researchers must be aware of ever-improving statistical techniques that account for (known) sequencing biases (e.g. Beissbarth and Speed 2004; Finotello et al. 2014; Kanehisa et al. 2008; Young et al. 2010; Zeeberg et al. 2003). Researchers must synthesize and use existing biological knowledge, often from multiple disciplines. Then, using a data-dense resource of whole-transcript sequencing in a post hoc directed examination of (a) target genes of known biological function (in a classical qPCR style approach) with (b) functional group analysis (e.g. gene enrichment data), great progress can be made in understanding genomic effects. However, we emphasize that it is essential to have a fundamental understanding of the biological processes of interest. That is not to say that whole-transcript studies are without merit; on the contrary-they are of great importance, especially when used as a library to be probed for explicit questions or to discover novel transcripts. However, we cannot stress enough the fundamental need to understand the biology under investigation.

\section{Summary}

We have presented and evaluated here a developmental control hypothesis for the regulation and expression of predatorinduced phenotypic plasticity. Predator-induced morphological plasticity is regulated by ligand dose-dependent juvenoid 
signalling in $D$. pulex, and, based on our review of arthropod developmental biology, this may be quite a conserved process. We suggest that this, and the logical extension to joint juvenoid/ecdysteroid regulation of life history, is a general mechanism underpinning predator-induced plasticity in arthropods.

First, where changes in life history and morphology are present, and drawing on decades of arthropod endocrinemoulting-morphology research, our eco-devo hypothesis (Sultan 2007) predicts the regulation of predator-induced changes in morphology by the juvenoid endocrine pathway and of life history by a combination of ecdysteroid and juvenoid pathways. Second, we have presented a small gene network dominated by nuclear receptors that are vital for arthropod development and can discriminate experimentally between juvenoid and ecdysteroid action. Third, our endocrine pathway hypothesis is easily linked to established developmental control mechanisms, facilitating further prediction about the physiological regulation of plasticity and potentially the physiological axis on which genetic variation in plasticity is manifest.

Our results confirm a central role of the crustacean juvenile hormone (methyl farnesoate) in the induction of morphological change in juvenile instars of $D$. pulex. We show that the juvenoid pathway, as predicted, appears to regulate induced morphological defences, the central induced phenotypic change early in life in D. pulex. We also show that the developmental control of the juvenoid response is centred on the titre of gene products driving the expression. Our results, drawn from the expression of highly conserved genes in one of the most central endocrine signalling systems in arthropods, are likely to be generalisable to other species. The results of this experiment complement previous research into abiotic forms of stress (see Table 1) and reveal juvenile hormone to be central to processing stress.

Acknowledgments The manuscript benefitted from comments by $\mathrm{T}$. Little, G. Frazer and several reviewers. Alison Blake, Butch Crabtree and Bo Bagshaw maintained the Daphnia populations used in this study. SRD, GAL and APB were supported by a grant from the Natural Environment Research Council, UK (NERC standard grant, NE/ D01244/).

Open Access This article is distributed under the terms of the Creative Commons Attribution License which permits any use, distribution, and reproduction in any medium, provided the original author(s) and the source are credited.

\section{References}

Abrams PA, Rowe L (1996) The effects of predation on the age and size of maturity of prey. Evolution 50:1052-1061

ASTM (2007) Standard guide for conducting acute toxicity tests on test materials with fishes, macro invertebrates, and amphibians (ASTM E729-96). ASTM International, West Conshohocken
Baldwin IT, Halitschke R, Kessler A, Schittko U (2001) Merging molecular and ecological approaches in plant-insect interactions. Curr Opin Plant Biol 4:351-358

Beckerman AP, Rodgers GM, Dennis SR (2010) The reaction norm of size and age at maturity under multiple predator risk. J Anim Ecol 79:1069-1076

Beissbarth T, Speed TP (2004) GOstat: find statistically over represented gene ontologies within a group of genes. Bioinformatics 20:1464-1465. doi:10.1093/bioinformatics/bth088

Beldade P, Brakefield PM (2002) The genetics and evo-devo of butterfly wing patterns. Nat Rev Genet 3:442-452

Benard MF (2004) Predator-induced phenotypic plasticity in organisms with complex life histories. Annu Rev Ecol Evol Syst 35:651-673. doi:10.1146/annurev.ecolsys.35.021004.11 2426

Berger EM, Dubrovsky EB (2005) Juvenile hormone molecular actions and interactions during development of Drosophila melanogaster. In: Litwack G (ed) Insect hormones, vol 73. Elsevier, Amsterdam, pp 175-215

Brakefield PM, Kesbeke F, Koch PB (1998) The regulation of phenotypic plasticity of eyespots in the butterfly Bicyclus anynana. Am Nat 152:853-860

Chang ES, Bruce MJ, Tamone SL (1993) Regulation of crustacean molting: a multi-hormone system. Am Zool 33:324-329

Colbourne JK et al (2011) The ecoresponsive genome of Daphnia pulex. Science 331:555-561. doi:10.1126/science.1197761

Cruz J, Martin D, Pascual N, Maestro JL, Piulachs MD, Belles X (2003) Quantity does matter. Juvenile hormone and the onset of vitellogenesis in the German cockroach. Insect Biochem Mol Biol 33:1219-1225

Davidowitz G, Nijhout HF (2004) The physiological basis of reaction norms: the interaction among growth rate, the duration of growth and body size. Integr Comp Biol 44:443-449

Dennis SR, Carter MJ, Hentley WT, Beckerman AP (2011) Phenotypic convergence along a gradient of predation risk. Proc $\mathrm{R}$ Soc B Biol Sci 278:1687-1696

Emlen DJ, Nijhout HF (1999) Hormonal control of male horn length dimorphism in the dung beetle Onthophagus taurus (Coleoptera: scarabaeidae). J Insect Physiol 45:45-53

Endo K, Kamata Y (1985) Hormonal control of seasonal morph determination in the small copper butterfly, Lycaena phlaeas daimio seitz. J Insect Physiol 31:701-706

Erezyilmaz DF (2011) The genetic and endocrine baisi for the evolution of metamorphosis in insects. In: Flatt T, Heyland A (eds) Mechanisms of life history evolution: the genetics and physiology of life history traits and trade-offs. Oxford University Press, Oxford, pp 56-71

Escriva H, Bertrand S, Laudet V (2004) The evolution of the nuclear receptor superfamily. Essays Biochem 40:11-26

Finotello $\mathrm{F}$ et al (2014) Reducing bias in RNA sequencing data: a novel approach to compute counts. BMC Bioinformatics 15. doi: 10.1186/1471-2105-15-s1-s7

Flatt T, Heyland A (2011) Mechanisms of life history evolution: the genetics and physiology of life history traits and trade-offs. Oxford University Press, Oxford

Gelman DB, Blackburn MB, Hu JS (2002) Timing and ecdysteroid regulation of the molt in last instar greenhouse whiteflies (Trialeurodes vaporariorum). J Insect Physiol 48:63-73

Hall BL (1999) Nuclear receptors and the hormonal regulation of Drosophila metamorphosis. Am Zool 39:714-721

Hammill E, Rogers A, Beckerman AP (2008) Costs, benefits and the evolution of inducible defences: a case study with Daphnia pulex. J Evol Biol 21:705-715. doi:10.1111/j.1420-9101.2008.01520.x

Hannas BR, LeBlanc GA (2010) Expression and ecdysteroid responsiveness of the nuclear receptors HR3 and E75 in the crustacean Daphnia magna. Mol Cell Endocrinol 315:208-218 
Hannas BR, Wang YH, Baldwin WS, Li YC, Wallace AD, LeBlanc GA (2010) Interactions of the crustacean nuclear receptors HR3 and E75 in the regulation of gene transcription. Gen Comp Endocrinol 167:268-278. doi:10.1016/j.ygcen.2010.03.025

Hardie J (1980) Juvenile hormone mimics the photoperiodic apterization of the alate gynopara of aphid, Aphis fabae. Nature 286:602-604

Hardie J, Baker FC, Jamieson GC, Lees AD, Schooley DA (1985) The identification of an aphid juvenile hormone, and its titer in relation to photoperiod. Physiol Entomol 10:297-302

Hartfelder K, Emlen DJ (2005) Endocrine control of insect polyphenism. Compr Mol Insect Sci 3:651-703

Hebert PDN (1978) Population biology of Daphnia (Crustacea, Daphnidae). Biol Rev Camb Philos Soc 53:387-426

Heyland A, Degnan S, Reitzel AM (2011) Emerging patterns in the regulation of evolution of marine inverterbrate settlement and metamorphosis. In: Flatt T, Heyland A (eds) Mechanisms of life history evolution: the genetics and physiology of life history traits and trade-offs. Oxford University Press, Oxford, pp 29-42

Hoverman JT, Relyea RA (2009) Survival trade-offs associated with inducible defences in snails: the roles of multiple predators and developmental plasticity. Funct Ecol 23:1179-1188

Jindra M, Riddiford LM (1996) Expression of ecdysteroid-regulated transcripts in the silk gland of the wax moth, Galleria mellonella. Dev Genes Evol 206:305-314

Kanehisa M et al (2008) KEGG for linking genomes to life and the environment. Nucl Acids Res 36:D480-D484. doi:10.1093/nar/ gkm882

King-Jones K, Thummel CS (2005) Nuclear receptors-a perspective from Drosophila. Nat Rev Genet 6:311-323

Laforsch C, Tollrian R (2004) Embryological aspects of inducible morphological defenses in Daphnia. J Morphol 262:701-707

Lambertsen CJ, Bunce PL, Drabkin DL, Schmidt CF (1952) Relationship of oxygen tension to hemoglobin oxygen saturation in the arterial blood of normal men. J Appl Physiol. 4:873-885

Lass S, Spaak P (2003) Chemically induced anti-predator defences in plankton: a review. Hydrobiologia 491:221-239

Laurila A, Pakkasmaa S, Crochet PA, Merila J (2002) Predatorinduced plasticity in early life history and morphology in two anuran amphibians. Oecologia 132:524-530. doi:10.1007/ s00442-002-0984-7

Lauss M, Visne I, Kriegner A, Ringner M, Jonsson G, Hoglund M (2013) Monitoring of technical variation in quantitative high-throughput datasets. Cancer Informatics 12:193-201. doi:10.4137/cin.s12862

Miyakawa H, Gotoh H, Sugimoto N, Miura T (2013) Effect of juvenoids on predator-induced polyphenism in the water flea, Daphnia pulex. J Exp Zool A Ecol Genet Physiol 319:440-450. doi:10.1002/jez.1807

Moczek AP, Emlen DJ (1999) Proximate determination of male horn dimorphism in the beetle Onthophagus taurus (Coleoptera: scarabaeidae. J Evol Biol 12:27-37

Naraki Y, Hiruta C, Tochinai S (2013) Identification of the precise kairomone-sensitive period and histological characterization of necktooth formation in predator-induced polyphenism in Daphnia pulex. Zoolog Sci 30:619-625. doi:10.2108/zsj.30.619

Nijhout HF (1999) Control mechanisms of polyphenic development in insects-in polyphenic development, environmental factors alter some aspects of development in an orderly and predictable way. Bioscience 49:181-192

Nijhout HF (2003a) The control of growth. Development 130:58635867. doi:10.1242/dev.00902

Nijhout HF (2003b) Development and evolution of adaptive polyphenisms. Evol Dev 5:9-18

Oda S, Kato Y, Watanabe H, Tatarazako N, Iguchi T (2011) Morphological changes in Daphnia galeata induced by a crustacean terpenoid hormone and its analog. Environ Toxicol Chem 30:232-238

Olmstead AW, Leblanc GA (2002) Juvenoid hormone methyl farnesoate is a sex determinant in the crustacean Daphnia magna. J Exp Zool 293:736-739

Olmstead AW, LeBlanc GA (2007) The environmental-endocrine basis of gynandromorphism (intersex) in a crustacean. Intern J Biol Sci 3:77-84

Oshlack A, Wakefield MJ (2009) Transcript length bias in RNAseq data confounds systems biology. Biol Direct 4. doi: 10.1186/1745-6150-4-14

Parejko K (1992) Embryology of Chaoborus-induced spines in Daphnia pulex. Hydrobiologia 231:77-84

Parthasarathy R, Palli SR (2009) Molecular analysis of juvenile hormone analog action in controlling the metamorphosis of the red flour beetle, Tribolium castaneum. Arch Insect Biochem Physiol 70:57-70

Peckarsky BL, McIntosh AR, Taylor BW, Dahl J (2002) Predator chemicals induce changes in mayfly life history traits: a wholestream manipulation. Ecology 83:612-618

Pigliucci M (2001) Phenotypic plasticity: beyond nature and nurture. The Johns Hopkins University Press, Baltimore

Priya TAJ, Li FH, Zhang JQ, Yang CJ, Xiang JH (2010) Molecular characterization of an ecdysone inducible gene E75 of Chinese shrimp Fenneropenaeus chinensis and elucidation of its role in molting by RNA interference. Comp Biochem Physiol B 156:149-157

Relyea RA (2001a) Morphological and behavioral plasticity of larval anurans in response to different predators. Ecology 82: 523-540

Relyea RA (2001b) The relationship between predation risk and antipredator responses in larval anurans. Ecology 82:541-554

Riddiford LM (1993) Hormone receptors and the regulation of insect metamorphosis. Receptor 3:203-209

Riddiford LM (2008) Juvenile hormone action: a 2007 perspective. J Insect Physiol 54:895-901

Riddiford LM, Hiruma K, Zhou XF, Nelson CA (2003) Insights into the molecular basis of the hormonal control of molting and metamorphosis from Manduca sexta and Drosophila melanogaster. Insect Biochem Mol Biol 33:1327-1338

Rider CV, Gorr TA, Olmstead AW, Wasilak BA, Leblanc GA (2005) Stress signaling: coregulation of hemoglobin and male sex determination through a terpenoid signaling pathway in a crustacean. $\mathrm{J}$ Exp Biol 208:15-23

Riessen HP (1999) Predator-induced life history shifts in Daphnia: a synthesis of studies using meta-analysis. Can J Fish Aquat Sci $56: 2487-2494$

Roff D (2002) Life history evolution. Sinauer Associates, Sunderland

Rountree DB, Nijhout HF (1995) Hormonal control of a seasonal polyphenism in Precis coenia (Lepidoptera, Nymphalidae). J Insect Physiol 41:987-992

Safranek L, Riddiford LM (1975) Biology of black larval mutant of tobacco hornworm, Manduca sexta. J Insect Physiol 21:1931-1938

Sawada H, Nakagoshi M, Reinhardt RK, Ziegler I, Koch PB (2002) Hormonal control of GTP cyclohydrolase I gene expression and enzyme activity during color pattern development in wings of Precis coenia. Insect Biochem Mol Biol 32:609-615

Siaussat D, Bozzolan F, Queguiner I, Porcheron P, Debernard S (2004) Effects of juvenile hormone on 20-hydroxyecdysone-inducible EcR, HR3, E75 gene expression in imaginal wing cells of Plodia interpunctella lepidoptera. Eur J Biochem 271:3017-3027

Soin $\mathrm{T}$ et al (2008) Juvenile hormone analogs do not affect directly the activity of the ecdysteroid receptor complex in insect culture cell lines. J Insect Physiol 54:429-438 
Sullivan AA, Thummel CS (2003) Temporal profiles of nuclear receptor gene expression reveal coordinate transcriptional responses during Drosophila development. Mol Endocrinol 17:2125-2137. doi:10.1210/me.2002-0430

Sultan SE (2007) Development in context: the timely emergence of eco-devo. Trends Ecol Evol 22:575-582

Suzuki Y, Nijhout HF (2006) Evolution of a polyphenism by genetic accommodation. Science 311:650-652. doi:10.1126/ science. 1118888

Tan A, Palli SR (2008) Edysone receptor isoforms play distinct roles in controlling molting and metamorphosis in the red flour beetle, Tribolium castaneum. Mol Cell Endocrinol 291:42-49

Tatarazako N, Oda S, Watanabe H, Morita M, Iguchi T (2003) Juvenile hormone agonists affect the occurrence of male Daphnia. Chemosphere 53:827-833. doi:10.1016/s0045-6535(03)00761-6

Teplitsky C, Plenet S, Joly P (2005) Costs and limits of dosage response to predation risk: to what extent can tadpoles invest in anti-predator morphology? Oecologia 145:364-370

Tollrian R (1995a) Chaoborus crystallinus predation on Daphnia pulex: can induced morphological changes balance effects of body size on vulnerability? Oecologia 101:151-155. doi:10.100 7/BF00317278

Tollrian R (1995b) Predator-induced morphological defenses-costs, life-history shifts, and maternal effects in Daphnia pulex. Ecology 76:1691-1705

Tollrian R, Dodson SI (1999) Predator induced defenses in cladocerans. In: Tollrian R, Harvell CD (eds) The ecology and evolution of inducible defenses. Princeton University Press, Princeton

Tollrian R, Harvell CD (1999) The ecology and evolution of inducible defences. Princeton University Press, Princeton

Tollrian R, Von Elert E (1994) Enrichment and purification of chaoborus kairomone from water-further steps toward its chemical characterization. Limnol Oceanogr 39:788-796

Truman JW, Riddiford LM (2007) The morphostatic actions of juvenile hormone. Insect Biochem Mol Biol 37:761-770
Truman JW, Riddiford LM, Safranek L (1973) Hormonal control of cuticle coloration in tobacco hornworm, Manduca sexta-basis of an ultrasensitive bioassay for juvenile hormone. J Insect Physiol 19:195-203

Tuller T (2014) Challenges and obstacles related to solving the codon bias riddles. Biochem Soc Trans 42:155-159. doi:10.1042/ bst20130095

Van Buskirk J (2002) A comparative test of the adaptive plasticity hypothesis: relationships between habitat and phenotype in anuran larvae. Am Nat 160:87-102

Weiss LC, Kruppert S, Laforsch C, Tollrian R (2012) Chaoborus and Gasterosteus anti-predator responses in Daphnia pulex are mediated by independent cholinergic and GABAergic neuronal signals. Plos One 7:e36879. doi:10.1371/journal.pone.0036879

Weston DJ, Gunter LE, Rogers A, Wullschleger SD (2008) Connecting genes, coexpression modules, and molecular signatures to environmental stress phenotypes in plants. BMC Syst Biol 216. doi:10.1186/1752-0509-2-16

Young MD, Wakefield MJ, Smyth GK, Oshlack A (2010) Gene ontology analysis for RNA-seq: accounting for selection bias. Genome Biol 11:R14. doi:10.1186/gb-2010-11-2-r14

Zeeberg BR et al (2003) GoMiner: a resource for biological interpretation of genomic and proteomic data. Genome Biol 4:R28. doi:10.1186/gb-2003-4-4-r28

Zera AJ, Bottsford J (2001) The endocrine-genetic basis of life-history variation: the relationship between the ecdysteroid titer and morph-specific reproduction in the wing-polymorphic cricket Gryllus firmus. Evolution 55:538-549

Zera AJ, Denno RF (1997) Physiology and ecology of dispersal polymorphism in insects. Annu Rev Entomol 42:207-230

Zhou XG, Tarver MR, Scharf ME (2007) Hexamerin-based regulation of juvenile hormone-dependent gene expression underlies phenotypic plasticity in a social insect. Development 134:601-610. doi:10.1242/dev.02755 